\title{
Microbiological changes in pregnancy with antenate death of fetus
}

\author{
Ihtiyarova Gulchehra, Kilicheva Vazira, Rozikova Dildora, \\ Tosheva Iroda, Hafizoda Dilnoza \\ Department of Obstetrics and Gynecology. Bukhara State Medical Institute, Uzbekistan.
}

\section{Email address:}

gulchehra.uzb@mail.ru (Ihtiyarova Gulchehra)

\section{To cite this article:}

Ihtiyarova Gulchehra, Kilicheva Vazira, Rozikova Dildora, Tosheva Iroda, Hafizoda Dilnoza. Microbiological changes in pregnancy with antenate death of fetus. Journal of research in health science. Vol. 1, No. 2, 2018, pp. 18-25. DOI 10.26739/2523-1243

\section{dol http://dx.doi.org/10.26739/2523-1243/-2018-1-2-3}

\begin{abstract}
The main cause of AFD in pregnant women is the microbial (infectious) factor.In this regard, the identification of representatives of coagulase negative staphylococci (CNS), coagulase positive staphylococci (CPS) and enterobacteria from the vagina of women is suggested as an additional diagnostic criterion for establishing the dysbiosis of this biotope and an additional unfavorable prognostic criterion for the development of ADF in pregnant women.The adverse microbial background is higher in the comparison group than in the main group.This must be taken into account when conducting and organizing medical and consultative care for pregnant women and parturients with a risk of developing ADF.

Despite significant successes in antenatal fetal protection, in some cases under the influence of various adverse factors, intrauterine death occurs at different gestation periods, which dictates the need to study the causes and early delivery of the woman.] 3,6,8]
\end{abstract}

Objective: To study microbiological changes in pregnant women with antenatal fetal death. To solve the problems, we examined pregnant women with antenatal fetal death (AFD). All examined pregnant women permanently resided in the Bukhara region of the Republic of Uzbekistan.

The materials of our work included the results of studies of 308 patients, of which the main group was 134 women $(43.5 \pm$
$2.8 \%$ ), a comparison group of 123 women $(39.9 \pm 2.8 \%)$. The control group included 51 patients $(16.6 \pm 2.1 \%)$ with the physiological course of pregnancy and childbirth giving birth to a live child.

In turn, the main group of women with AFD $(n=134)$ was divided into 2 subgroups: subgroup 1a - 50 pregnant women, who were induced by Mifepristone and Glandin; subgroup $1 \mathrm{~b}$ - 84 pregnant women, induction of labor 
was carried out with the drug Mifepristone and misoprostol $25 \mathrm{mg}$ on the background of immunocorrective therapy.

Pregnant women with AFD included in the comparison group retrospective group $(\mathrm{n}=123)$ for 2005-2009, depending on the method of induction, as well as the inductor itself, were divided into 3 subgroups: subgroup 2a-39 pregnant women who were induced by induction of delivery with the introduction of uterotonics ; subgroup $2 \mathrm{~b}-44$ pregnant women, whose induction of the injection was administered (PGR?) 2-alpha (Enzaprost); subgroup 2B - 40 pregnant women who were born without induction, pregnant with the control group $(\mathrm{n}=51)$ with the physiological course of pregnancy and childbirth giving birth to a live child, stimulation of labor was not carried out.

The criteria for inclusion in the main group and the comparison group were: the established diagnosis of AFD; age of pregnant women under 40; indications for the induction of labor; availability of informed consent of pregnant women to participate in scientific research. One of the main causes of antenatal fetal death (AFD) in pregnant women is infection of the fetus in various ways, including through the placenta.

In this regard, we were interested in knowing the state of the microbiocenosis of the lower and upper parts of the birth canal of women in the dynamics of the course of pregnancy and childbirth.

Bacteriological studies of biological materials from the vagina, placenta and amniotic fluid were conducted in pregnant women and women with AFD, as well as women with a physiological pregnancy and giving birth to a live child. The studies were conducted in the dynamics of the course of pregnancy - in the III-trimester, before childbirth (or before induction) and after childbirth (or after stillbirth).

It was established that the normocenosis in the study dynamics - in the III-trimester, before delivery (or before induction) and after childbirth (or after stillbirth) - gradually decreases in all the examined groups, except for the control group. A particularly marked decrease in this parameter was observed in the comparison group. The results of normocenosis obtained in the examined groups were significantly low in relation to the parameters of the control group $(\mathrm{P}<0.01)$. If in the III-trimester the normocenosis index in the control group was $76.5 \pm 5.9 \%$, in the la subgroup this parameter was $30.0 \pm 6.5 \%$, in $1 b$ subgroup $-39.3 \pm 5.3 \%$, in 2 a subgroup $-28.2 \pm 3.4 \%$, in the $2 b$ subgroup - 38.6 $\pm 7.3 \%$ and $I n$ the subgroup, $32.5 \pm 7.4 \%$ $(\mathrm{P}<0.001)$. Although significant differences were found between the control group and the tested groups, no significant differences were observed between this group (1a and $1 \mathrm{~b}$ of the subgroup) and the comparison group (2a, 2b, 2c subgroups) in this parameter $(\mathrm{P}>0.05)$.

If in the dynamics of the study in the control group, normocenosis rates were constantly increasing (before and after birth, respectively, $88.2 \pm 4.5 \%$ and 98.0 $\pm 1.9 \%)$, in the examined groups, these indicators significantly decreased ( $\mathrm{P}$ $<0.05)$. When describing the state of normal vaginal microflora in the dynamics of the study, the following features were established: firstly, in the control group, the normocenosis parameters gradually increase significantly in the study dynamics, and in the main group and in the comparison group significantly 
Ihtiyarova Gulchehra, Kilicheva Vazira, Rozikova Dildora, Tosheva Iroda, Hafizoda Dilnoza.

Microbiological changes in pregnancy with antenate death of fetus.

decrease; secondly, the parameters of normocenosis in the group examined were 2.5-9.8 times significantly less than in the control group; thirdly, between all the subgroups of the study (the main group and the comparison group), close to each other tend to change the parameters of normocenosis.

In accordance with changes in normocenosis indicators, a different number of pregnant and parturient women were found with dysbiotic changes in the microflora of the lower sections of the genital tract. If in the control group in women with a physiological pregnancy and the birth of a live child the dysbiosis of the vagina was noted in single cases in the III-trimester $(5.9 \pm 3.3 \%, \mathrm{n}=3)$ and were not observed before and after childbirth, then in the main group and the comparison group, dysbiotic changes were found in a significantly larger number of subjects. A clear pattern of increasing the percentage of vaginal dysbiosis in the study dynamics was determined (except for subgroup 1a). In most cases, especially in subgroups $2 b$ and 2c, these differences were statistically significant $(\mathrm{P}<0.05)$. In the main group and the comparison group, the indices of dysbiosis were from $38.6 \pm 7.3 \%$ (in the III trimester in the $2 \mathrm{~b}$ subgroup) to $70.0 \pm 7.3 \%$ (after birth in the $2 b$ subgroup).

The next parameter of worthy attention was the presence of pathogenic microorganisms, including trichomonads. If in the control group pathogenic microorganisms were found in isolated cases and only in the III-trimester $(9.8 \pm$ $4.2 \%, \mathrm{n}=5$ ), in other surveyed groups pathogenic microorganisms were identified in a large number of examined. They were sown most often in the comparison group than in the main group.

Thus, the analysis of the results of the study of the state of the normal vaginal microflora shows that the dynamics of the study determined the following features: First, the normocenosis indices in the control group were 2.5-9.8 times higher than in the main group and in the group comparison; Secondly, in the control group, the parameters of normocenosis increased in the dynamics of the study and after the delivery reached almost the maximum level, while in the main group and the comparison group, the normocenosis indexes decreased in dynamics; thirdly, there was no significant difference in the normocenosis indices in the main group and in the comparison group; Fourth, there is a high incidence of vaginal dysbiosis in the main group and the comparison group, which significantly differ from the control data up to several dozen times; Fifth, pathogenic and conditionally pathogenic microorganisms (including Trichomonas), characterized by high massiveness of excretion, were planted in the vagina of pregnant women and parturients of the main group and the comparison group, and practically no pathogens were found in the control group; Sixth, the percentage of seeding of pathogens in all groups gradually increased in the study dynamics, reaching a maximum before delivery, which is not typical for the control group; Seventh, a close, inverse relationship between detectability of normocenosis, vaginal dysbiosis and the presence of pathogens in the lower genital tract was detected.

These facts point to a lack of functioning of the indigenous representatives of the normal microflora, lack of self-cleaning 
process of the lower genital tract and high transient colonization and pathogenic agents of the biotope in pregnant women and women in labor before delivery.

After establishing the fact that in pregnant women with AFD, dysbiotic events occur significantly more than in healthy women who gave birth to a live child, we studied the frequency of detection of strains of microorganisms colonizing the lower section of the genital tract in pregnant women with an AFD of the main group, a comparison group and a control group.

It was found that in pregnant women with the AFD of the main group, the indigenous representative of the normal vaginal microflora, Lactobacillus spp, is significantly less $(\mathrm{P}<0.001)$ in the women of the main group than in the control group $-40.0 \pm 6.9 \%$ in the $1 \mathrm{a}$ subgroup $-44.1 \pm 5.4 \%$ and in the control $-88.2 \pm 4.5 \%$ (Table 4.2).

Practically identical results, but with less intensity, results were also obtained from the sowing capacity of Bifidobacteriumspp. If in $1 \mathrm{a}$ and $1 \mathrm{~b}$ subgroups the percentage of sowing of this microorganism was $32.0 \pm 6.6 \%$ and 39.3 $\pm 5.3 \%$, respectively, in the control group this index reached $60.8 \pm 6.8 \%(\mathrm{P}<0.001)$.

The incidence of asporagene anaerobes (Peptostreptococcuspp and Bacteroidesspp) is significantly less than the above representatives of the indigenous microflora of the vagina. By their detectability, this regularity was determined, as in the sowing of previous microorganisms.

A different picture of the microbial landscape of the vagina was observed in the analysis of the seeding of pathogenic and conditionally pathogenic microorganisms (CPM). Most often in pregnant women with AFD (main group) coagulase-negative staphylococci (CNS) - S.epidermidis, the percentage of sowing was significantly higher than other pathogenic and CPM in the compared groups. Detectability of S.epidermidis, respectively, by 2.7 and 3.6 times greater than in control $(\mathrm{P}<0.001)$. Similar results were obtained for coagulase-positive staphylococcus (CPS) S. aureus, as well as Escherichiaspp, Candidaspp and T. vaginalis $(\mathrm{P}<0.001)$.

Thus, in pregnant women with AFD, a significant decrease in the indigenous representatives of the normal microflora of the vagina (Lactobacillusspp and Bifidobacteriumspp) was established in relation to the control from 1.5 to 2.2 times. Similar results were obtained for the seeding of Bacteroidesspp and Peptostreptococcuspp, but the percentage of Streptococcuspp (group D) and di phtheriadid not practically change and remained at the level of the control group. It is established that the seeding of pathogenic and CPM is significantly more than in the control group. This fact indicates that colonization of the mucosa by pathogenic and CPM (gram-positive cats, gram-negative bacteria, microscopic fungi and trichomonads) leads to a disturbance of the balance between the indigenous and facultative microorganisms of the vaginal microbiocenosis. From the foregoing it follows that the main cause of AFD in pregnant women is the microbial (infectious) factor. In this regard, the identification of representatives of CNS, CPS and enterobacteria from the vagina of women is suggested as an additional diagnostic criterion for establishing the dysbiosis of this biotope and an additional unfavorable prognostic criterion for the 
development of AFD in pregnant women. In contrast to the main group, a significant increase in bacteroides and diphtheriawith respect to the control group $(\mathrm{P}<0.05)$ was noted in women with AFD and the comparison group. Another difference is that the seeding of peptostreptococci was at the level of the control group, that is, no statistically significant differences were observed ( $\mathrm{P}>0.001)$.

Thus, the microbial spectrum of the sown strains from the vagina in pregnant women with AFD of the comparison group was almost at the level of the main group, which differed in some imbalance in the seeding of bacteroides, diphtheria and streptococcus in group D. In addition, an increased level of germination of CNS, CPS, Escherichia, Candide and trichomonads. From the foregoing it follows that all the studied indicators in the comparison group were worse than in the main group. This fact indicates that an unfavorable microbial (infectious) background is higher in the comparison group than in the main group. This must be taken into account when conducting and organizing medical and consultative care for pregnant women and parturient women with the risk of developing AFD.

\section{References}

1. Abildinova G.Zh., Bayanova MF, Nagymtaeva A.A. Genetic aspects of reproductive health disorders // J.Doktor axborotnomasi.-2009.-№3.-S.. 264-265

2. Abdullaeva MA Prevention of intrauterine infection of the fetus in pregnant women with chlamydiaureaplasma infection // News of dermatovenerology and reproductive health.-2008.-No.1-P.12-14.

3. Abdurahmanova F.M. Neuro-exchange-endocrine disorders in girls with the pathology of the development of the reproductive system // Pediatrics.-2003.-No.1-S-69-72.

4. Abu Sarah Khalil Ahmad, Lakatosh V.P. The state of the indices of the humoral immune system in patients with women on urogenital mycoplasmosis // Collection of scientific works of the Association of Obstetricians-Gynecologists of Ukraine .- Kiev .- "Intermed" .- 2007.- P. 5-9.

5. Amirova $\mathrm{Zh}$. S. The system of cytokines in pregnant women with a persistent and recurrent threat of abortion // J. Newsletter of new medical technologies. - 2006. - T. 13.- № 4. - P. 66-67.

7. Alekseev LP, Boldyreva M.N. Revision of the concept of the role of HLA antigens in the physiology and pathology of the reproductive process // Zh. Physiology and pathology of the immune system. 2004.-T.8, No. 1.-P.44-51.

8. Dolgushina N.V., Makatsaria A.D. Viral infections in pregnant women. A guide for doctors. M: TriadX 2004.

9.Qiu, Q. Role of pro- IGF-II processing by proproteinconvertase 4 in human placental development / Q. Qiu, A. Basak, M. Mbikai // Proc. Natl. Acad. Sci USA. 2005. - Vol. 102.-No. 31.-P. 11047-11052. 